\title{
Progress of Comprehensive Nursing on the Prevention of Postoperative DVT of Gynecologic Tumor
}

\author{
Shaohua Wang', Jianli Jiang2, Xiaobing $\mathrm{Lu}^{3}$ \\ ${ }^{1}$ The Second People's Hospital of Yichang, Yichang, China \\ ${ }^{2}$ The First People's Hospital of Yichang, Yichang, China \\ ${ }^{3}$ The Institute of Mechanics, Chinese Academy of Sciences, Beijing, China \\ Email:xblu@imech.ac.cn
}

How to cite this paper: Wang, S.H., Jiang, J.L. and Lu, X.B. (2020) Progress of Comprehensive Nursing on the Prevention of Postoperative DVT of Gynecologic Tumor. Advances in Reproductive Sciences, $\mathbf{8}$, 201-208.

https://doi.org/10.4236/arsci.2020.84017

Received: September 15, 2020

Accepted: October 13, 2020

Published: October 16, 2020

Copyright $\odot 2020$ by author(s) and Scientific Research Publishing Inc. This work is licensed under the Creative Commons Attribution International License (CC BY 4.0).

http://creativecommons.org/licenses/by/4.0/

\begin{abstract}
Deep vein thrombosis (DVT) is the second reason of death due to tumor. In order to find more effective methods for prevention of DVT due to gynecologic tumor, the advances of study and practice in this aspect should be summarized. As an important and effective measure for prevention of DVT, the comprehensive nursing is to be analyzed carefully. Lots of practices show that proper nursing measures can prevent effectively the occurrence of postoperative DVT. Virchow thought that DVT is caused by three factors: injury of venous wall, slowed blood flow and hypercoagulability. Thus, the prevention measures are presented on the factors such as application of sequential decompression elastic socks, intraoperative intervention etc. Clinical nursing pathway is shown to be very effective method recently. More effective measures and related facilities for prevention of DVT should be built in the future. Physical and psychological methods should be combined in the nursing of DVT.
\end{abstract}

\section{Keywords}

Deep Vein Thrombosis, Tumor, Nursing, Prevention of DVT

\section{Introduction}

Vein thrombosis (VTE) means the abnormal blood clotting in the vena cava, which affects the reflux of blood in vein. VTE often occurs in deep vein, especially lower limbs to form deep vein thrombosis (DVT) or lower deep vein thrombosis (LDVT). If DVT is not found in time, pulmonary embolism (PE) can occur due to the thrombus shedding. The incidence rate of DVT is about $7 \%$ 
- 45\% after gynecological surgery [1]. It is reported that VDT and PE are the second reason of death due to tumor [2] [3].

Patients with gynecologic tumor are the high-risk population of DVT. But it is often misdiagnosed and treated late. Proper nursing measures can prevent effectively the occurrence of postoperative DVT.

The aim of this paper is to summarize the comprehensive nursing on the prevention of postoperative DVT due to gynecologic tumor for deeper study on more effective methods in the future. The mechanism of occurrence of DVT and the main prevention measures by comprehensive nursing are discussed. 24 literatures are chosen in this paper. 3 of them are chosen for introduction of DVT, 9 on the study of mechanism of occurrence of DVT, 18 on the study and practice of prevention measures.

\section{Mechanism of Occurrence}

In the middle of 19 century, Virchow presented a theory that DVT is caused by three factors: injury of venous wall, slowed blood flow and hypercoagulability [4]. The occurrence of DVT is sketched in Figure 1. This theory has been validated after near 100 years clinical application. It is thought that the possibility occurring DVT of tumor patients is 4 - 7 times of general population. Gynecological tumor patients have higher danger of DVT because of the special physiological and anatomical structure and surgical treatment [5].

The factors and mechanism related with the occurrence of DVT are mainly as follows: 1) factor of tumor, malignant tumor cells can secrete cell factor to reduce anticoagulant material and increase coagulant, which provides the pathological basis of local thrombus [6] [7]; 2) surgical factors, for example, the radical hysterectomy is the main operation mode of endometrial cancer and cervical cancer. Patients must be preoperative fasting and drinking, so the organism is easy to dehydrate and the blood is easy to concentrate. In addition, the affected range of radical hysterectomy is large because the pelvic lymphadenectomy is often
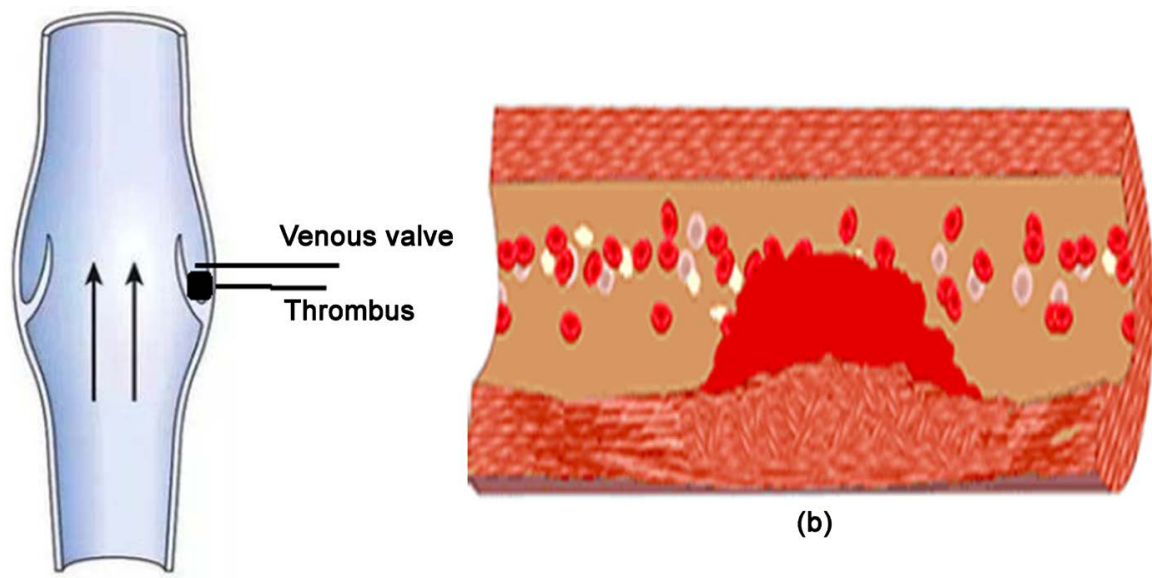

(b)

(a)

Figure 1. Sketch of occurrence of DVT. (a) Occurrence at the back of venous valve due to slow flood flow; (b) Occurrence due to injury of vascular wall. 
processed. The vascular wall and the surrounding tissues are easy to be damaged during operation. Meanwhile, the operation time and anesthesia time are long and easy to make the muscles of the lower limbs flabby and the surrounding muscles of the lower limbs become flabby and the veins enlarge. Long-term lying in bed after operation can cause urinary retention, blockage of venous return. All these factors can cause DVT [8] [9] [10]. 3) anatomic factors: Veins in female pelvic are dense. The vein wall is thin and has not valve, which make women easier to form venous blood stasis [11]. 4) chemotherapy: If the patients adapt chemotherapy as the successive treatment after radical hysterectomy, the possibility of occurring DVT is 2 - 6 times of the general population. The reason is that chemotherapy increases the content of procoagulant protein and the platelet activation, meanwhile restrain the fibrinolysis process [12].

\section{Prevention Measures}

\subsection{Application of Sequential Decompression Elastic Socks}

The effects are obvious for the prevention of DVT by applying sequential decompression elastic socks and air wave pressure treatment system.

The theory of the sequential decompression elastic socks is to form progressively declining pressure by putting the stocks with elastic pressure on the foot, ankle and shank. The pressure at the ankle is about $18 \mathrm{mmHg}, 14 \mathrm{mmHg}$ at the middle leg. The declined pressure can force the blood return and relief the pain of lower limbs.

The treatment system of air wave pressure inflates and bleeds periodically to the multicavity bags of pressure leg cover. The pressure squeezes the legs uniformly and regularly from distal to proximal. The pressure can excite the deep muscles, blood vessel and lymph gland of lower limbs to increase the oxygen carrying capacity of blood and improve microcirculation, which can effectively decrease the pain of lower limbs and prevent DVT [13].

\subsection{Intraoperative Intervention}

Try best to succeed at the first time of establishing venous access before operation and avoid the central venous catheterization of lower extremity. During intraoperative fixing position, the limbs should not be fixed too tight or too abducted. The patients with high risk factors should use the elastic socks or other equipment to promote venous return. The operation should be finished as soon as possible to decrease vascular injury and blood loss.

Postoperative intervention: The patient should be turn over every two hours in the first 6 hours after operation. Then the patient should start activity. The family members should help the patient to massage the limbs and perform joint activities. For those with small wound and good recovery, they'd better to start off-bed activity. The activity can promote the blood circulation and improve the venous return of lower limbs. The patients having abdominal distension, urinary retention or intestinal obstruction should be treated as soon as possible to pre- 
vent the risk of DVT [14].

It is shown that the effects of preventive care on guarding against postoperative DVT in gynecological malignant tumor. The surgical effect and the patient satisfaction are high. Not only the hospital stays can be shortened, but also the leg circumference growth can be controlled. So the possibility of DVT can be reduced greatly [15].

Chemotherapy is the common measure to treat malignant tumor. Administration is mainly through veins. The treatment often lasts half a year or over one year. The multiple administration through peripheral vein can cause local phlebitis, arteriosclerosis atresia, permanent injury of blood vessels and surrounding tissues because of the repeat excitation of drugs and puncture in a long treatment circle. The exosmose of blister medicine can also cause inflammatory response or even massive necrosis of tissues.

As an alternative method of infusion by peripheral vein puncture, peripherally inserted central catheter (PICC) can avoid the above problems. PICC is first used to provide the parenteral nutrition for the patients in severe condition in 1975. Considering the simple operation, high safety and prevention of blood vessel injury due to repeat puncture, PICC is widely used in the intravenous chemotherapy of patients with malignant tumor.

The cacumen of PICC can arrive directly to the superior vena cava, which can reduce the vessel injury due to irritant chemotherapy drugs. However, PICC-related venous thrombosis often occurs because of the hypercoagulable state of tumor patients and the nausea and vomiting, fatigue and weakness, long-term bedridden, limb swelling etc. after chemotherapy. If the thrombus shedding causes pulmonary embolism, the patients are in danger of death [12].

Therefore, prevention of PICC-related DVT is very important in the treatment of chemotherapy. Nursing intervention is an effective method to reduce the occurrence of PICC-related DVT.

The risk factors of PICC-related DVT can be divided into four types [16]-[23]: 1) Patients' general conditions such as age, sex, obesity, daily activity, history of smoking, scale of blood vessels. 2) Conditions of diseases and treatment such as surgical history, history of chemotherapy, history of radiotherapy, history of previous thrombosis, history of PICC, usage of erythropoietic drugs and antiplatelet drugs, preventive or therapeutic anticoagulation, diseases of hypertension, diabete, coronary heart disease, chronic obstructive pulmonary disease etc. 3) Conditions of catheterization such as puncture times, position of catheter tip, position of puncture, type and scale of catheter etc. 4) Conditions of complication related with catheter such as infection due to catheter, catheter heterotopic etc.

The effective and safe exercise mode of upper limb for the patients with PICC is that the patients start exercise after 24 hours of PICC and hold a ball 30 times continuously after each meal to increase the venous return.

Physical intervention and drug intervention are main measures to prevent DVT. For PICC-related DVT, physical intervention is better than drug interven- 
tion because preventive use of anticoagulants cannot reduce the incidence rate of PICC-relate DVT, but early exercise of upper limbs can prevent this type of DVT effectively. In recent years, many studies on the exercise of upper limbs are processed considering the mode, frequency, opportunity. For example, the exercise with gripping device is mainly studied [24] [25]. It is shown that the exercise with gripping device is not suitable for most tumor patients. Clenching is a kind of perfect exercise to prevent PICC-related DVT. The amplitude and force of motion of this exercise are moderate and so can avoid the occurrence of complication related with PICC [26].

Either intraoperative use of intermittent pneumatic pressure pump (IPPP) alone or combined use of gradient compression elastic socks (GCES) and IPPP can effectively prevent the occurrence of DVT after digestive tract cancer surgery. However, use of GCES combined with IPPP cannot significantly reduce the incidence of deep venous thrombosis in patients undergoing gastrointestinal cancer surgery relative to use of IPPP alone, but can significantly improve the blood flow velocity of external iliac vein, deep femoral vein and popliteal vein, $\mathrm{D}$-dimer and FIB indexes after digestive tract cancer surgery [27].

Acupoint stimulation can improve the swelling degree of affected limbs, reduce the skin temperature of affected limbs, reduce Homan sign and Neuhof sign, and improve the coagulation index, improve lower extremity blood flow and reduce the occurrence of DVT after meningioma [28].

\subsection{Clinical Nursing Pathway}

Clinical nursing pathway (CNP) can provide a series of regular and standardized nursing interventions to reduce DVT.

CNP is a kind of nursing mode made for the patients according to the standard nursing plan of a special disease or operation. Every conventional nursing plan is listed out in figures or tables, which can help nursing staff to carry out effectively [29].

Hua Lu's study shows that CNP can effectively reduce the risk of DVT after radical hysterectomy. CNP can optimize the nursing procedure, reduce the hospital stays and improve the satisfaction of patients. Detection of plasma FIB and DD is benefit to the early diagnosis of DVT after radical hysterectomy. The changes of D-dime, fibrinogen and platelet can help to judge if the patients are in the state of hypercoagulability and if nursing intervention should be strengthened [30].

\section{Conclusions}

Comprehensive nursing on the postoperative DVT of gynecologic tumor is summarized in this paper. The mechanism of occurrence and the main prevention measures are discussed.

Proper nursing measures to effectively prevent the occurrence of postoperative DVT are very important as it is one of the main reasons of death due to tu- 
mor. Because the main factors causing DVT are thought as three factors: injury of venous wall, slowed blood flow and hypercoagulability, the presented prevention measures are mainly on these factors such as application of sequential decompression elastic socks, intraoperative intervention etc. Clinical nursing pathway is shown to be a very effective method recently.

In the future, more effective measures and related facilities for prevention of DVT should be built. Physical and psychological methods should be combined in the nursing of DVT. Because the physical conditions, disease type, operation type and economic situation of each patient are different, the nursing measures should be more personalized. New physical prevention tools similar to air bag for repeat pressurization should be developed, especially those that can affect deep blood flow without physical injury. The combination of Traditional Chinese Medicine and West Medicine for prevention of DVT is also a very promising direction.

\section{Conflicts of Interest}

The authors declare no conflicts of interest regarding the publication of this paper.

\section{References}

[1] Mao, Me., Qiao, Y.H. and Guo, R.X. (2010) Etiology and Prevention of Deep Venous Thrombosis of Lower Limbs after Gynecological Surgery. Shandong Medical Journal, 50, 115-116. (in Chinese)

[2] Lan, X.C. and Feng, Z.R. (2002) The Cardiovascular Surgery. 2nd Edition, People's Medical Publishing House, Beijing, 1255-1333. (in Chinese)

[3] Rickles, F.R. and Levine, M.N. (2001) Epideminology of Thrombosis in Cancer. Acta Haematologica, 106, 6-12. https://doi.org/10.1159/000046583

[4] Zhang, B.G. (2006) Several Problems on the Treatment and Prognosis of Deep Venous Thrombosis of the Lower Limbs. Chinese Journal of General Surgery, 21, 81. (in Chinese)

[5] Khorana, A.A., Kuderer, N.M., Culakova, E., et al. (2008) Development and Validation of a Predictive Model for Chemotherapy-Associate Thrombosis. Blood, 111, 4902-4907. https://doi.org/10.1182/blood-2007-10-116327

[6] Yokota, N., Koizume, S., Miyagi, E., et al. (2009) Self Production of Tissue Factor-Coagulation Factor VII Complex by Ovarian Cancer Cells. British Journal of Cancer, 101, 2023-2029.

[7] Wang, Y.T. and Zhao, H.R. (2011) Progress on the Study of Malignant Tumor and Venous Thromboembolism. Journal of Modern Oncology, 19, 1028-1031. (in Chinese)

[8] Jin, L., Shen, K., Lang, J.H., et al. (1999) Diagnosis, Treatment and Prevention of the Complication of Deep Venous Thrombosis after Gynecologic Oncology Surgery. Chinese Journal of Obstetrics and Gynecology, 34, 488-490. (in Chinese)

[9] Sun, Z. (2003) Investigation on the Related Factors of the Lower Deep Venous Thrombosis after Hys-Terectomy. Chinese Nursing Research, 17, 890-892. (in Chinese)

[10] Wang, H.Y., Li, D.F., Li, Y.Z., et al. (2003) Clinical Analysis of 24 Cases with Leg 
Deep Venous Thrombosis after Operation for Gynecologic Tumors. Chinese Journal of Practical Gynecology and Obstetrics, 19, 108-109. (in Chinese)

[11] Blom, J.W., Vanderschoot, J.P., Oostindier, M.J., et al. (2006) Incidence of Venousthrombosis in a Large Cohort of 66329 Cancer Patients: Results of a Record Linkage Study. Thrombosis and Haemostasis, 4, 529-535. https://doi.org/10.1111/j.1538-7836.2006.01804.x

[12] Haddad, T.C. and Greeno, E.W. (2006) Chemotherapy-Induced Thrombosis. Thrombosis Research, 118, 555-568. https://doi.org/10.1016/j.thromres.2005.10.015

[13] Chen, Z.Y., Xu, J., Dong, S.H., et al. (2016) Effect Observation of Comprehensive Nursing Intervention in Preventing the Deep Venous Thrombosis of Lower Extremity of cervical Cancer after Operation. Chinese Nursing Training, 31, 1710-1712.

[14] You, Y.Q. (2019) Effect of Preventive Nursing on Preventing Deep Venous Thrombosis of Lower Limbs in Perioperative Period of Gynecological Tumor Operation. Guide of China Medicine, 17, 233-234. (In Chinese)

[15] Xu, L. (2019) Analysis of the Effect of Nursing Intervention on Lower Extremity Deep Vein Thrombosis after Gynecological Malignant Tumor Operation. Chinese Community Doctors, 35, 171-173. (In Chinese)

[16] Bob, L., Lobo, P.D., Georgeta, V., et al. (2009) Risk of Venous Thromboembolism in Hospitalized Patients with Peripherally Inserted Central Catheters. Journal of Hos pital Medicine, 4, 417-422. https://doi.org/10.1002/jhm.442

[17] Verso, M., Agnelli, G., Kamphuisen, P.W., et al. (2008) Risk Factors for Upper Limb Deep Vein Thrombosis Associated with the Use of Central Vein Catheter in Cancer Patients. Internal \& Emergency Medicine, 3, 117-122. https://doi.org/10.1007/s11739-008-0125-3

[18] Tran, H., Arellano, M., Chamsuddin, A., et al. (2010) Deep Venous Thromboses in Patients with Hematological Malignancies after Peripherally Inserted Central Venous Catheters. Leuk Lymphoma, 51, 1473-1477. https://doi.org/10.3109/10428194.2010.481065

[19] Gorski, L.A., Hagle, M.E. and Bierman, S. (2015) Intermittently Delivered IV Medication and PH: Reevaluating the Evidence. Journal of Infusion Nursing, 38, 27-46. https://doi.org/10.1097/NAN.0000000000000081

[20] Lee, A.Y.Y., Levine, M.N., Butler, G., et al. (2006) Incidence, Risk Factors and Outcomes of Catheter-Related Thrombosis in Adult Patients with Cancers. Journal of Clinical Oncology, 24, 1404-1408. https://doi.org/10.1200/JCO.2005.03.5600

[21] Cheng, K.O., Venkatesh, S.K., Lau, G.B., et al. (2010) Prospective Randomized Comparative Evaluation of Proximal Valve Polyurethane and Distal Valve Silicone Peripherally Inserted Central Catheters. Journal of Vascular \& Interventional Radiology, 21, 1191-1196. https://doi.org/10.1016/j.jvir.2010.04.020

[22] Caine, G.J., Stonelake, P.S., Rea, D., et al. (2003) Coagulopathic complications in Breast Cancer. Cancer, 98, 1578-1586.

[23] Yu, R. and Chen, L.F. (2016) Risk Factors of Venous Thromboembolism Associated with Peripherally Inserted Central Catheters among Cancer Patients: A Meta-Analysis. Chinese Nursing Management, 16, 738-743.

[24] Mei, S.J., Yu, J. and Yang, L.H. (2013) Effect of Fisting Activity at Different Frequencies on Hemodynamics of the Vein with PICC Insertion. Journal of China Clinic Medical Imaging, 24, 798-800

[25] Zhang, M., Fang, X.X., Li, M., et al. (2014) Effects of Fisting Activity on the Hemo- 
dynamics of Upper Veins. Chinese Journal of Nursing, 49, 1325-1329.

[26] Hui, H. (2018) Research on Evidence-Based Nursing Intervention Strategies for Preventing PICC-Related Venous Thrombosis. Thesis for Master Degree, Medical School of Chinese PLA, Chongqing.

[27] Zhao, Y.L. (2019) Effect of Intraoperative Physical Intervention on Prevention of Deep Venous Thrombosis of Lower Limbs in Patients with Digestive Tract Tumors. Thesis for Master Degree, Shanxi University of Chinese Medicine, Taiyuan.

[28] Chen, X. (2019) Effect of Acupoint Massage on Deep Venous Thrombosis in Patients with Meningioma after Operation. Thesis for Master Degree, Qingdao University, Qingdao.

[29] Luo, L. (2010) Research Progress on Detection and Clinical Application of D-Dimer. Sichuan Medical Journal, 10, 1562-1563.

[30] Hua, L. (2014) Application of Nursing Clinical Pathway on Prevention of Lower Deep Venous Thrombosis in Patients Undergoing Radical Hysterectomy. Thesis for Master Degree, Chongqing Medical University, Chongqing. 